\title{
colaboraciones
}

\section{EL CONTROL DE CALIDAD EN LAS CONSTRUCCIONES DE LA DIRECCION GENERAL DE CORREOS Y TELECOMUNICACION}

\author{
Ignacio MORILLA ABAD, \\ Dr. Ingeniero de Caminos \\ 073-13
}

El presente artículo es una ampliación y actualización de la comunicación presentada por el autor al Congreso de Control de Calidad en Construcción de Turín celebrado en abril de 1982 que contenía datos hasta 1981. Es una aportación de informaciones para cuantificar la rentabilidad de los procesos de control de calidad y contribuir así a la mayor aplicación de estos métodos. El autor, Ignacio Morilla, Subdirector General de Obras e Instalaciones de la Dirección General de Correos y Telecomunicación, hasta enero de 1983, describe aqui las acciones llevadas a cabo en los Edificios de Comunicaciones en 1980, 81 y 82 $y$ los beneficios económicos y de servicio que ha producido el Control de Calidad.

\section{Introducción}

La finalidad del presente artículo es la de aportar datos a la rentabilidad de una politica de control de calidad en la edificación.

El principal obstáculo que se presenta, a nuestro juicio, para la implantación del control de calidad en España, es que se sigue considerando por muchos sectores como un gasto adicional a la obra y no como una inversión rentable a corto y largo plazo.

Por ello pensamos que el camino más eficaz para que un sector industrial aplique el control de calidad, es convencer a los responsables económicos de su rentabilidad efectuando estadísticas y estudios que cuantifiquen los beneficios obtenidos con su aplicación.

Estos principios que se basan en nuestra propia experiencia, se apoyan en las conclusiones N. ${ }^{\circ} 10$

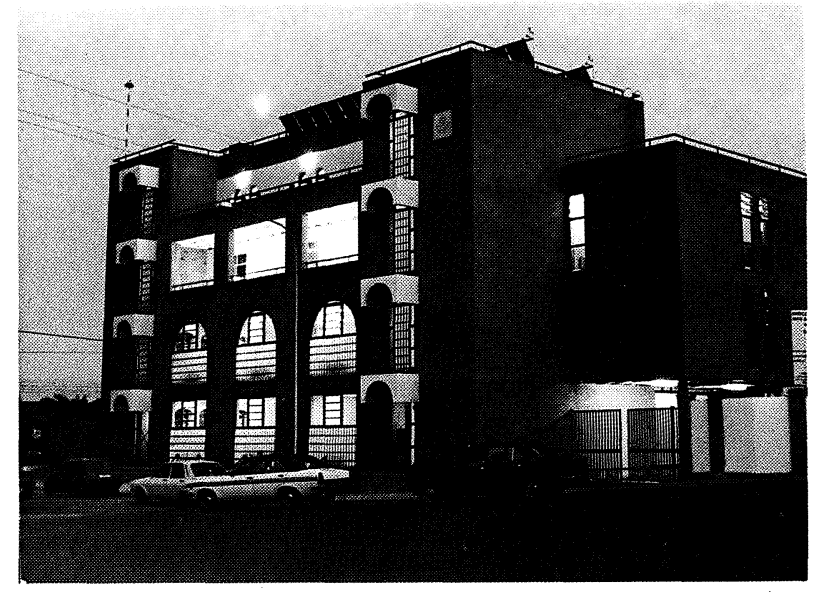

Pabellón postal de Jerez. Fachada principal. (Arquitecto Juan Salabert).

del Coloquio de 1976 y N. ${ }^{\circ} 8$ del Coloquio de 1979 de la Sección de Construcción de la Organización Europea de Control de Calidad (EOQC), que decian respectivamente:

«Recomendar a toda persona relacionada con el proceso constructivo la determinación de los gastos que se derivan de una falta de control. Los que de una manera errónea crean que el control es un lujo se sorprenderán ante la comparación de estos gastos, siempre elevados, con los que corresponden al establecimiento de un sistema de control."

"Estudiar y precisar los niveles de control a exigir a nivel de proyecto, ejecución y conservación, respecto a la amplitud de los riesgos, coste global de la construcción y duración prevista de su vida útil».

2. Construcción de edificios en la Dirección General de Correos y Telecomunicación. Aspectos Generales.

La Dirección General de Correos y Telecomunicación utiliza una extensa red de edificios y locales en toda España, cuyo número se acerca a 2.500 y que en su mayoria han sido construidos o adaptados en los años 1920 a 1940 respondiendo a unas necesiciades ya superadas. Hoy día esta infraestructura resulta insuficiente e inadecuada y desde el año 1979 se está tratando de adaptar a la nueva organización de las Comunicaciones que requiere unos métodos de trabajo más racionales. 


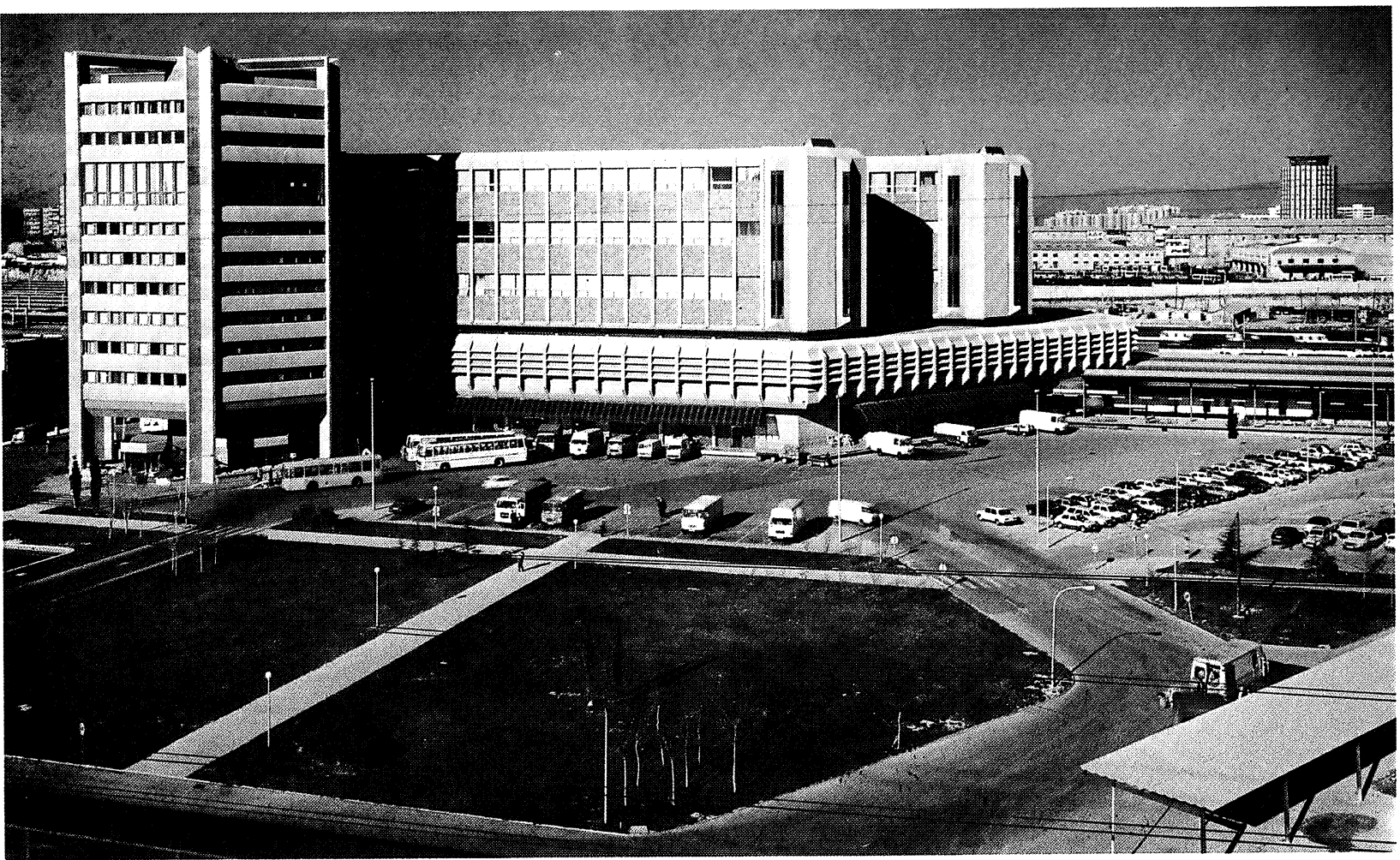

Centro de clasificación postal de Chamartín, Madrid. (Arquitecto José Luis González Cruz).

En lo que a edificios se refiere la politica que se ha adoptado es la de conservar y reformar los antiguos edificios situados en lugares céntricos y frecuentemente de valor artístico, adaptándolos a servicios burocráticos y administrativos y de atención al público, transfiriendo las funciones técnicas o trabajos que admiten mecanización a edificios especializados. Igualmente se ha pretendido acercar los servicios lo más posible a los usuarios y mejorar la calidad de servicio y condiciones de trabajo de los funcionarios.

Esta política de realizaciones iniciada a principios de 1980 (Fig. 1) se traduce en la práctica en:

a) Nuevos edificios para centrales-télex y de telegrafía pública situados en puntos de fácil conexión a redes existentes o futuras.

b) Pabellones postales para instalación de maquinaria de clasificación automática del correo y mecanizado del manejo de cargas situados en estaciones ferroviarias, de autobuses o terminales aéreos.

c) Desconcentración de carterias en grandes núcleos urbanos para reducir tiempos de entrega a los usuarios.

d) Reforma de edificios antiguos para adaptarlos a tareas administrativas.

e) Creación de Sucursales de Comunicaciones en barrios periféricos para mejorar la calidad del servicio y la atención al público. f) Integración de Oficinas de Correos y de Telecomunicación en pequeños núcleos de población.

g) Construcción de viviendas para funcionarios.

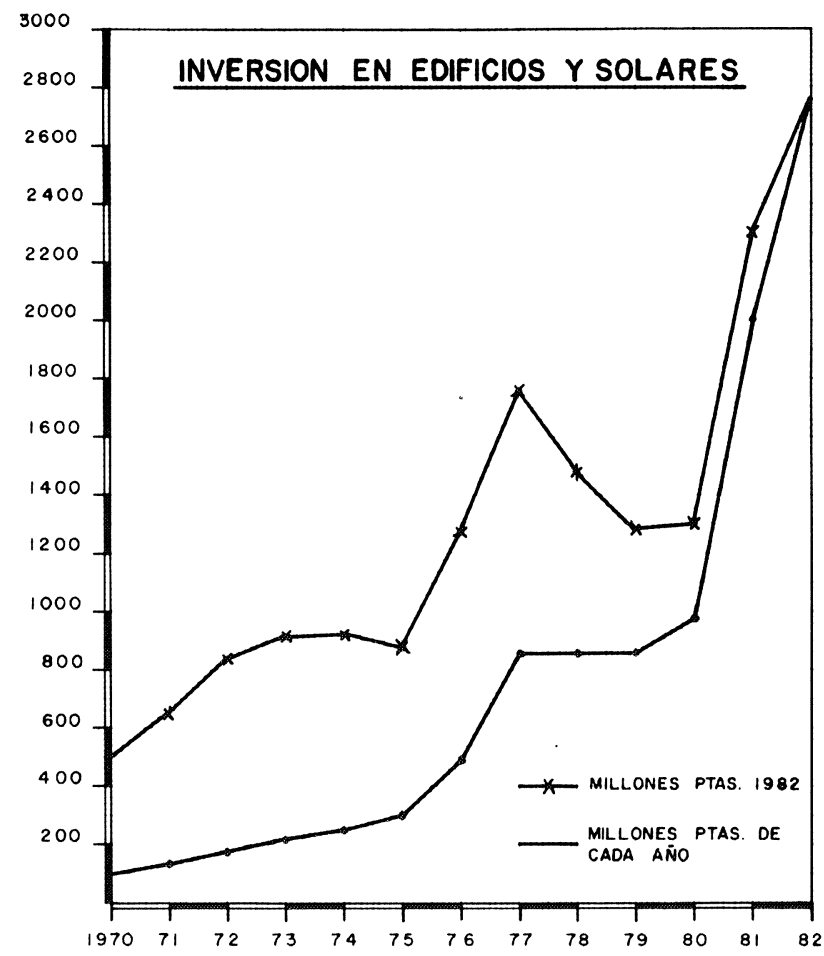

Fig. 1 
h) Racionalización del uso de almacenes y talleres mediante un plan nacional de aprovechamiento, al máximo, de sus recursos en función de situación y superficie.

Todas estas acciones encaminadas a modernizar la Infraestructura inmobiliaria han requerido el replanteamiento de la política de inversiones, incrementándose de manera notable los presupuestos de construcción y de adquisición de solares pero incidiendo de una forma acusada en la política de conservación de edificios y locales.

3. La problemática de la conservación del patrimonio inmobiliario. Nueva organización de los servicios de conservación.

El valor del patrimonio inmobiliario de la D.G.C. y T. en 1981 es de 32.073 millones de pesetas, y alcanzará la cifra de 72.125 millones de pesetas en 1991 (a pesetas de 1982) si se cumplen las previsiones de inversión previstas (Fig. 6). Las cantidades dedicadas a conservación en los últimos años han sido decrecientes tanto respecto al valor del patrimonio como en cantidades valoradas en pesetas de 1982 (Figs. 2-3-4-5).

La política de restricción del gasto público hace que las cantidades destinadas a gastos corrientes de utilización y conservación de edificios sean cada vez menos importantes, lo que obliga a afrontar el problema de la conservación de edificios con una nueva politica de distribución de las cantidades presupuestadas para la infraestructura inmobiliaria.

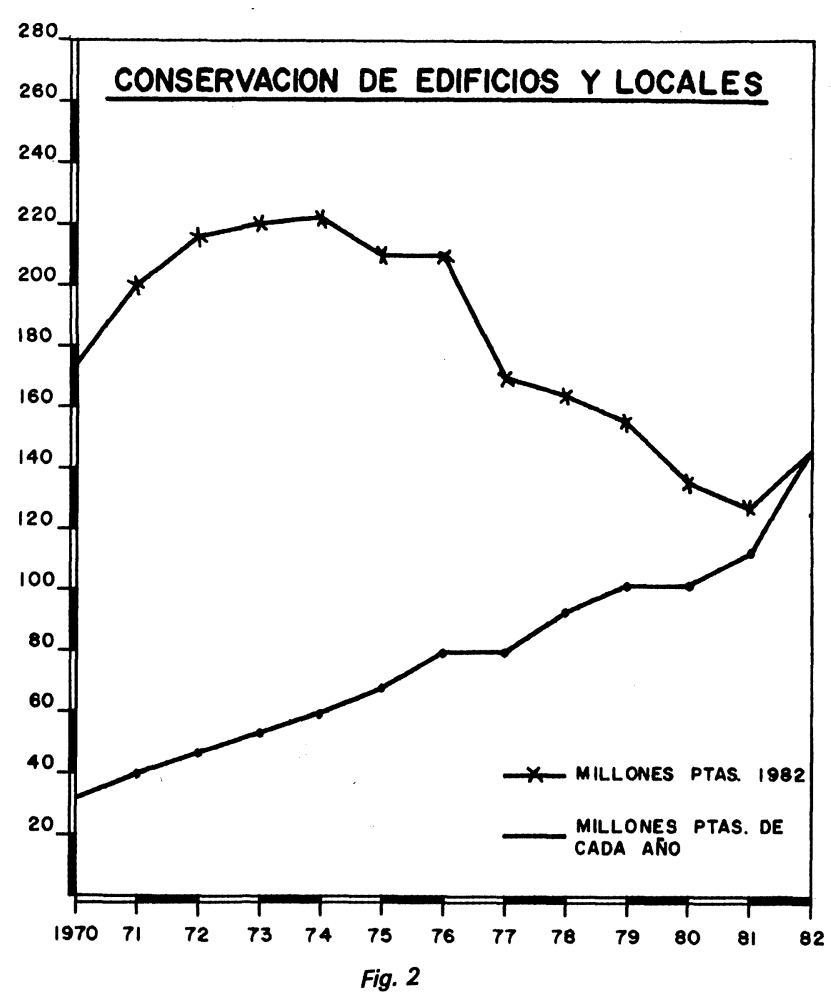

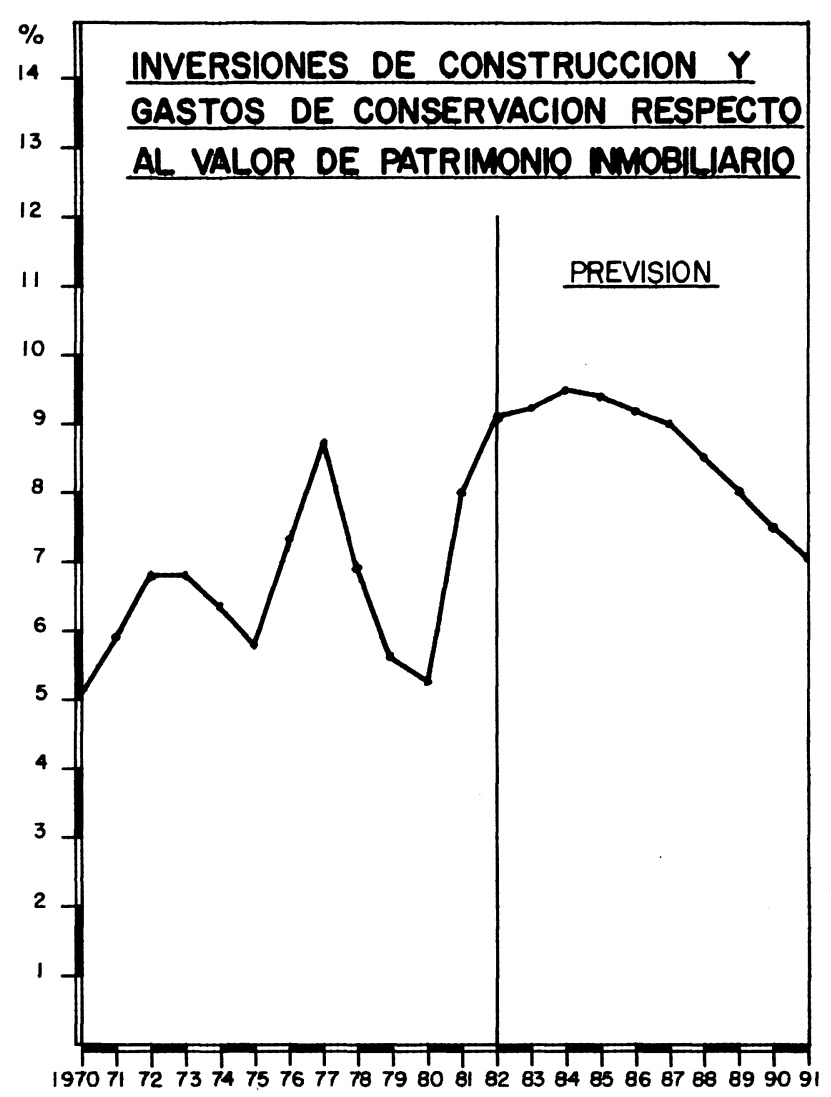

Fig. 3

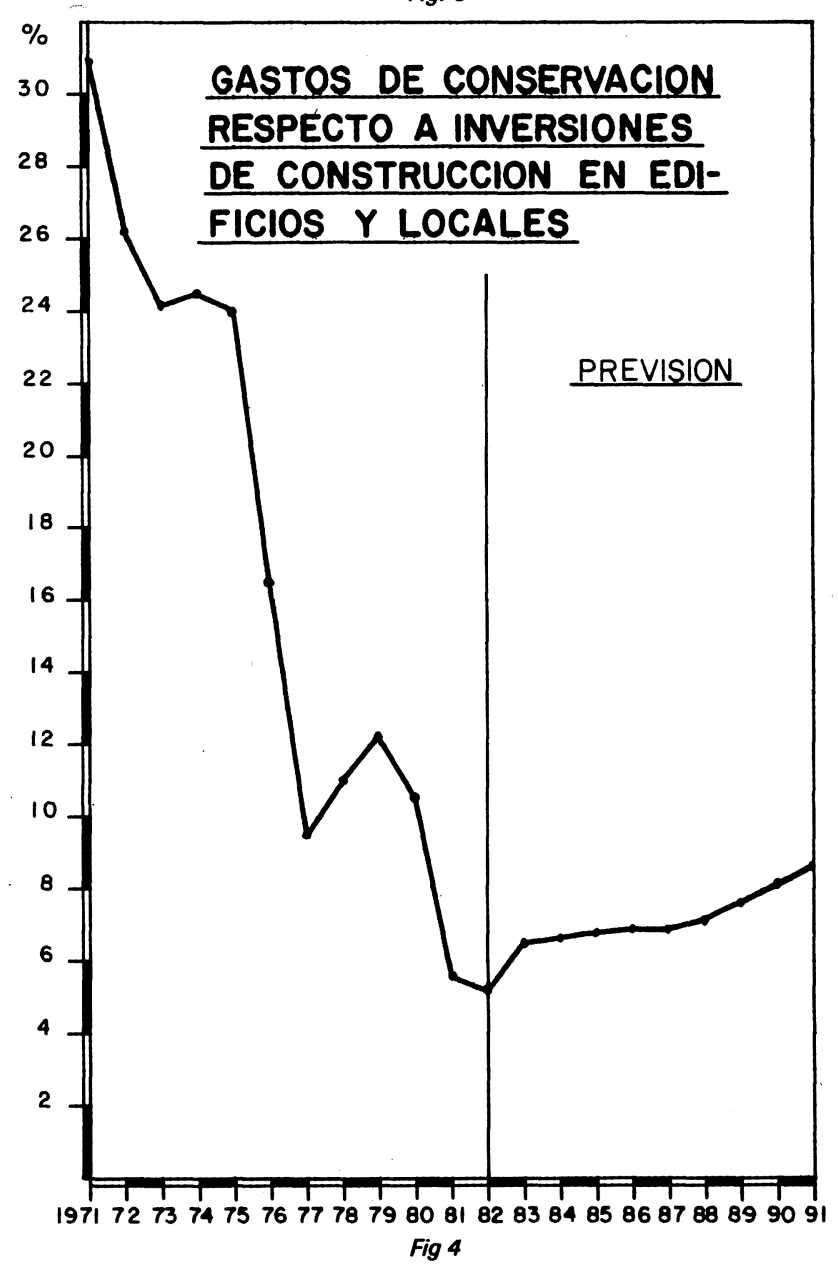




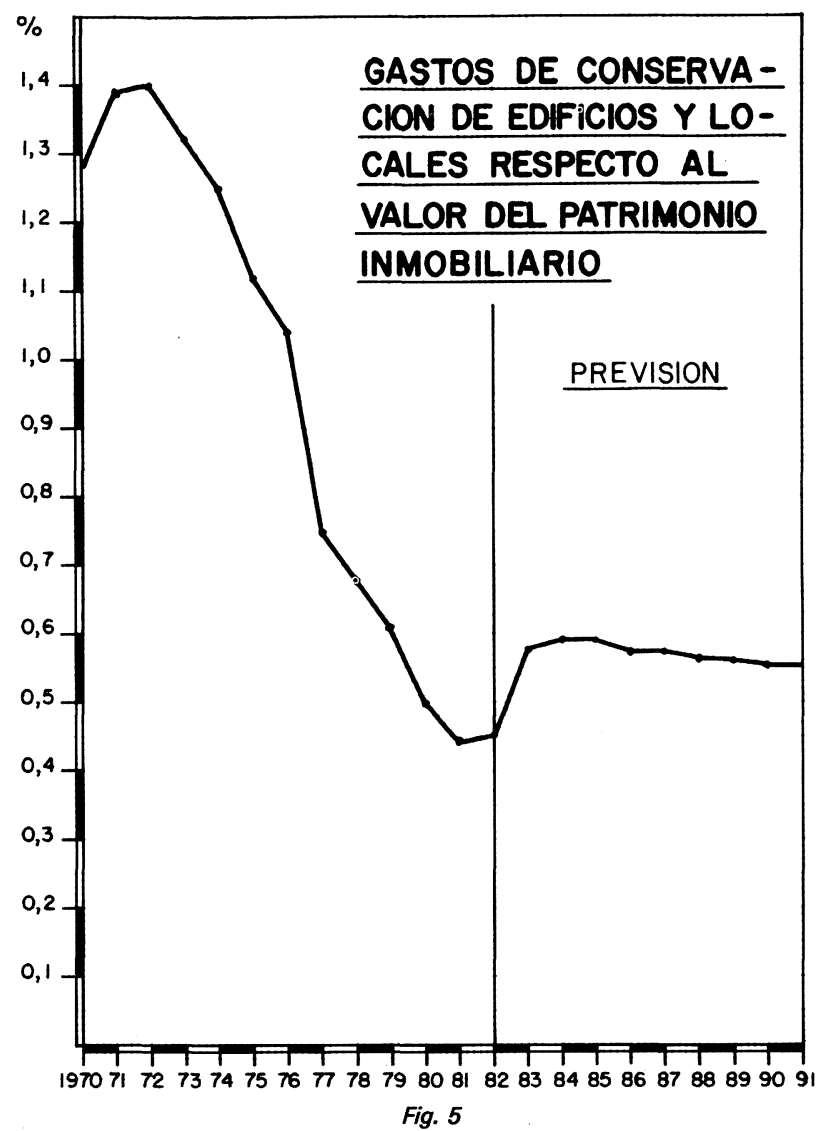

Las medidas que se han adoptado han sido las siguientes:

a) Unificación en un solo organismo gestor, la Subdirección General de Obras e Instalaciones, de la utilización de los presupuestos destinados a construcción y conservación con lo que se puede realizar una politica eficaz.

b) Crear, dentro de esta Subdirección, equipos de conservación de edificios, repartidos en todas las provincias, que realizan por sí mismos o gestionan la conservación de inmuebles. Actúan de una manera coordinada con el personal dedicado a conservación de instalaciones de telecomunicación o electromecánicas por lo que el aprovechamiento de recursos humanos y materiales es creciente.

c) Puesta en marcha de planes que inciden directa 0 indirectamente en la reducción de gastos de conservación de edificios por mejor utilización de equipos humanos o materiales como:

- Plan de ahorro energético (alumbrado, fuerza, calefacción, refrigeración, aislamientos, energías alternativas, etc.).

- Racionalización de transportes interiores y exteriores en los edificios.

- Automatización de las operaciones de clasificación y mecanización del correo, con ahorro de espacios.

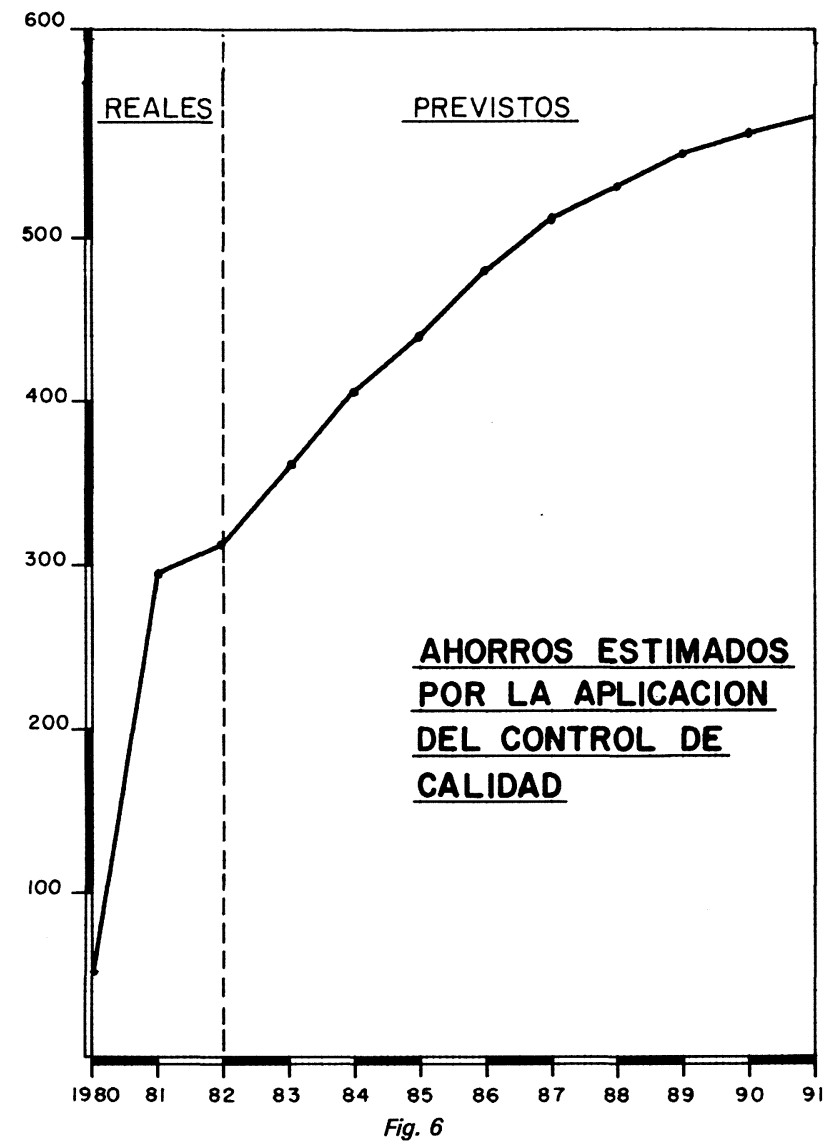

- Automatización creciente de sistemas de transmisión con gran ahorro de espacio en salas de aparatos, centrales de conmutación y transmisión.

- Utilización racional de maquinaria menor, como carretillas, canceladoras, atadoras, contenedores, máquinas de ventanilla, etcétera, con el consiguiente ahorro en gastos de conservación o consumos.

Todos estos planes tienen incidencia en la reducción de gastos de conservación y explotación de los edificios, pero el más importante de todos en cuanto a ahorro económico es el de la aplicación de un Sistema de Control de Calidad que permite economizar grandes cantidades de dinero en la construcción y, sobre todo, en la conservación futura de los inmuebles.

\section{La rentabilidad de una construccion de calidad en los edificios de Comunicaciones.}

El control de calidad aplicado a una extensa red de edificios, en adaptaciones o nuevas construcciones, es particularmente rentable en un futuro próximo en lo que se refiere a conservación, ya que la eficacia de los equipos de conservación es muy dudosa cuando hay que atender a numerosos problemas y éstos disminuyen mucho cuando las obras se han hecho con un sistema de control de calidad. Estos equipos se irán convirtiendo con el 
tiempo en grupos de trabajo de conservación preventiva en lugar de grupos de trabajo de reparaciones $o$ arreglo de averías.

La aplicación de los sistemas de control de calidad antes de la realización del proyecto permite hacer de éste con una mayor exactitud, reduciendo coeficientes de seguridad en la estimación de la mayoración, de la acciones que intervienen en el cálculo de estructura y cimientos. Por otra parte, los estudios previos en terrenos con ensayos e informes geotécnicos han permitido en muchos casos ahorros considerables en el proyecto de cimentaciones, si se comparan los costes previstos en el anteproyecto con los que han resultado en el proyecto. Los cálculos efectuados en un conjunto de proyectos han permitido estimar los ahorros en construcción en 50 millones de pesetas, en 1980 y calcular para 1981 una economia de 292 millones (Fig. 7). En el periodo de 10 años se estima que pueden economizarse más de $4.700 \mathrm{mi}-$ llones de pesetas de 1982 en los proyectos de construcción, aproximadamente un $10 \%$ del presupuesto de estos 10 años, todo ello a pesetas de 1982. (Fig. 7).

Sin embargo, el ahorro del $10 \%$ previsto en los gastos de construcción si ha podido valorarse con exactitud, en 1981 , controlando 118 proyectos que suponen:

- Un $11,47 \%$ de los proyectos realizados.

- Un 42,72\% del presupuesto total.

El presupuesto de control de calidad supuso una media de $1,19 \%$ del presupuesto y ha producido un ahorro calculado del $15,20 \%$, superior al $10 \%$ previsto en un $5 \%$.

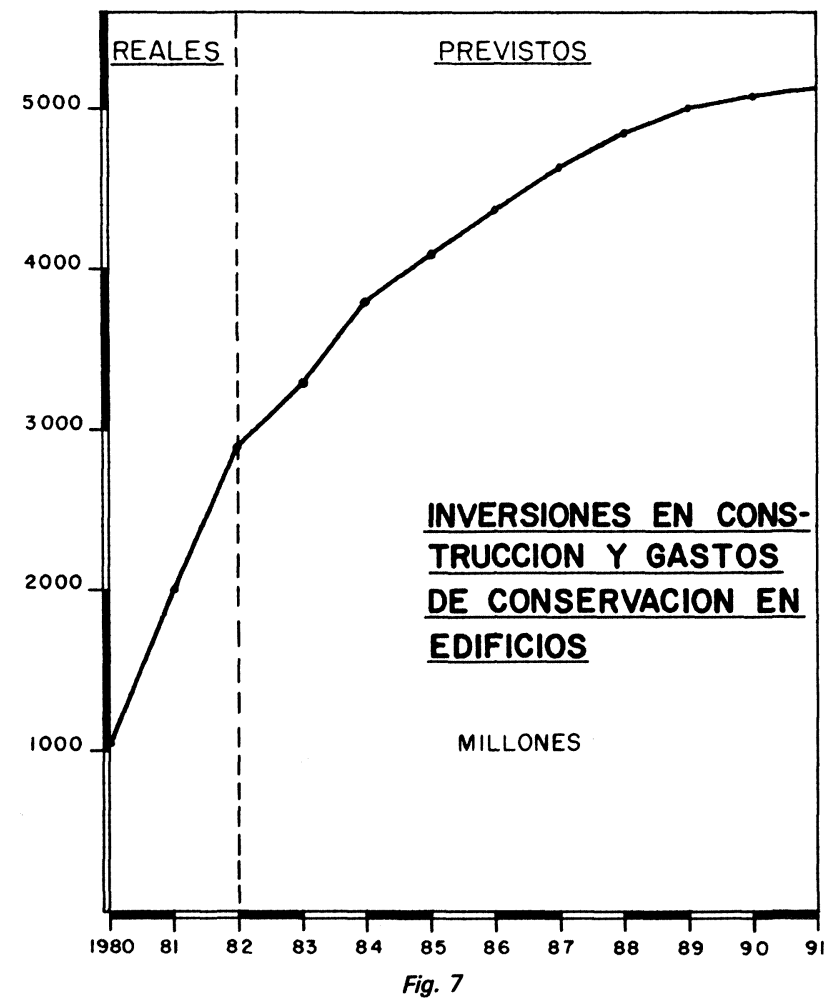

Dividiendo los proyectos en cuatro grupos, los datos obtenidos han sido los siguientes:

- En los proyectos de 0 a 5 millones de pesetas:

El presupuesto de control de calidad suponia un $0,625 \%$ y el ahorro estimado un 3,33\% del presupuesto de obra.

- En los proyectos de 5 a 20 millones de pesetas:

El presupuesto de control de calidad supone un $1,508 \%$ y el ahorro estimado un $\mathbf{8 , 7 3} \%$ del presupuesto de obra.

- En los proyectos de 20 a 100 millones de pesetas:

El presupuesto de control de calidad supone un $1,442 \%$ y el ahorro estimado un $11,65 \%$ del presupuesto de obra.

- En los proyectos de más de 100 millones de pesetas:

El presupuesto de control de calidad supone un $1,249 \%$ y el ahorro estimado un $17,75 \%$ del presupuesto de obra.

De aqui se deduce que el control de calidad apenas produce ahorro en la construcción de obras menores de 5 millones de pesetas, aunque sí lo producirá en su conservación.

El presupuesto de control de calidad es comparativamente más elevado en los proyectos de 5 a 20 millones de pesetas.

El ahorro producido por el control de calidad, es tanto más elevado en porcentaje, cuanto mayor es el presupuesto del proyecto.

Conclusiones todas ellas que corroboran lo que cabria esperar lógicamente de las mismas.

En estas estimaciones no están incluidas, por ser difícilmente valorables, las horas perdidas por las obras que se realizan en locales abiertos al público y que reducen el rendimiento de los funcionarios y producen molestias al público. De haberse estimado, el ahorro por un adecuado control de calidad habria sido mayor. Igualmente en obras programadas en su ejecución y en las que se ejerce un control de la calidad de su ejecución está demostrado que se reducen los plazos ya que se detectan a tiempo problemas que en caso contrario tardan más tiempo en resolverse.

En lo que se refiere a la reducción de gastos de conservación se han estimado 37 millones de pesetas para 1982 y hasta 100 en 1991 , con un total de 712 millones de pesetas (de 1982 en este periodo de 10 años) lo que significa un ahorro on conservación del $25 \%$ del presupuesto destinado a este fin en dicho periodo. Debido a que las can- 
tidades destinadas a conservación son más modestas, el total de la reducción es menos importante que en el capitulo de inversiones, pero más significativa desde el punto de vista que permite cumplir el objetivo general de reducción de gastos corrientes y posibilita la mejor utilización de los equipos de conservación.

Para estimar con mayor exactitud los ahorros en gastos de conservación, que se producen por la implantación del control de calidad, se han creado en la Dirección General de Correos y Telecomunicación unos "cuadernos de gastos" por cada edificio o local en donde se apuntan todos los derivados de la conservación, permitiendo comparar año por año, y por cada conjunto de edificios del mismo tipo, los gastos que se producen en los que han tenido control de calidad, respecto de los edificios que no lo han tenido. En 1982 se ha hecho la primera estimación que corrobora las cifras expresadas anteriormente, pero son necesarias correcciones de fecha y mayor serie estadistica para asegurar las previsiones.

La suma de ahorro económico en construcción y conservación varía de 314 millones de pesetas en 1982, a 570 millones de pesetas en 1991, con un total de 4.739 millones de pesetas (de 1981) de economia en 10 años. (Fig. 7).

En cuanto a los costes de este Control de Calidad, ya tenidos en cuenta en las estimaciones de ahorro anteriores, se han valorado previamente de una forma global para un conjunto de proyectostipo, como adaptación de locales, construcción de pabellones postales, nuevos edificios, oficinas, sucursales, habiéndose llegado a unas cotas máximas de porcentaje respecto a presupuestos que son las siguientes:

a) Estudios de investigación geotécnica previos al proyecto.

\section{Presupuestos:}

- mayor de 100 millones de Ptas., de 0,60\% a $1,40 \%$

- de 20 a 100 millones de Ptas., de 1,00\% a $2,10 \%$

- de 5 a 20 millones de Ptas., de 1,90\% a $7,30 \%$

(Los porcentajes más altos se refieren a proyectos de presupuesto bajo en terrenos muy difíciles).

b) Control de calidad mediante ensayos durante la construcción.

\section{Presupuestos:}

- mayor de 100 millones de Ptas., de 0,90\% a $1,20 \%$

- de 20 a 100 millones de Ptas., de 0,95\% a $2,20 \%$
- de 5 a 20 millones de Ptas., de 1,00\%

5. Aplicación del control de calidad a los Edificios de Comunicaciones.

La aplicación del control de calidad se realiza mediante las siguientes actuaciones:

a) Estudios geotécnicos previos para el proyecto de cimientos en estructuras importantes 0 zonas conflictivas, según unas directrices establecidas en la "Guía para la aplicación del Control de Calidad en los Edificios de Comunicaciones" en la que se determinan tres niveles de ensayos, imprescindibles, necesarios y convenientes, especificando el número y tipo de ensayos, así como su presupuesto. Estos ensayos se concretan para seis tipos de suelos: rocas duras, rocas blandas, gravas y suelos con partículas gruesas, arenas y suelos arenosos, arcillas de baja compresibilidad $y$ arcillas de alta compresibilidad, que se definen a efectos de aplicación de esta Guia. Todos estos grupos de ensayos se distribuyen a su vez en tres grandes conjuntos, según que el presupuesto del proyecto sea de 5 a $20 \mathrm{mi}$ llones de pesetas, de 20 a 100 o mayor de 100 . Asi pues, la "Guía" determina para cada tipo de edificio, tipo de suelo o nivel de control, la lista de ensayos a realizar y su presupuesto.

b) Ensayos de control de calidad durante la construcción según unos niveles establecidos en la "Guía" para cada tipo de edificio (de 5 a 20 , de 20 a 100 o más de 100 millones de pesetas), grado de control (ensayos imprescindibles, necesarios y convénientes) y unidades principales de la obra.

En la citada Guia se relacionan y presupuestan ensayos para:

\section{Acondicionamiento del terreno}

- Terraplenes, Desmontes, Drenajes.

\section{Cimentaciones}

- Zapatas; Pilones; Pantallas; Inyecciones...

\section{Estructuras}

- Metálicas; de Hormigón; Mixtas; Pretensadas; Forjados de viguetas, macizos y aligerados; Fábrica de ladrillo, de piedra, de bloques...

\section{Fachadas}

- Carpinterias metálicas, de madera, muros de paneles; Cerramientos de ladrillo; Celosias; Barandillas...

\section{Instalaciones}

- Calefacción; Electricidad; Aire acondicionado; Fontaneria; Ascensores; Gas... 


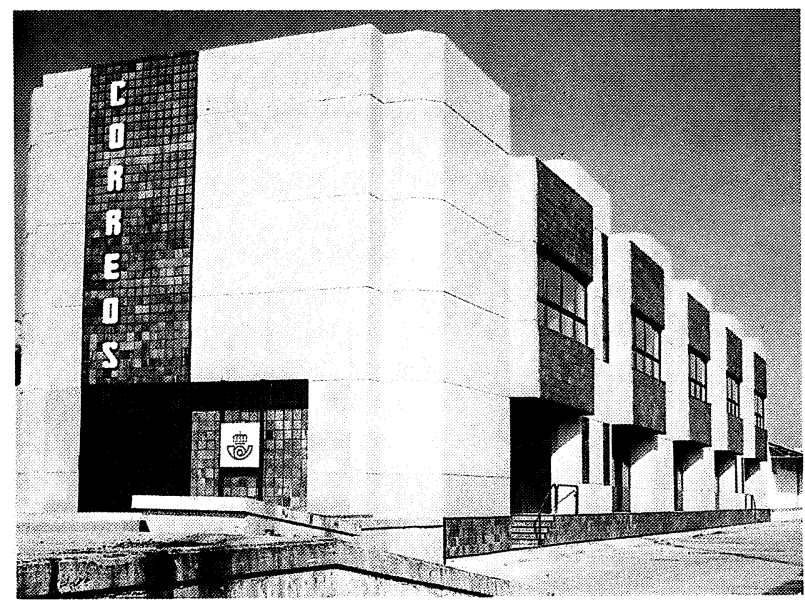

Centro de Clasificación postal de Santander. Estación de ferrocarril. (Arquitecto Antonio Mora).

\section{Particiones}

- Mamparas de acero, aluminio, madera, cristal, tabiques de ladrillo, prefabricados...

\section{Cubiertas y lucernarios}

- De fibrocemento, metálicos, de teja de pizarra, de cemento, de plástico, de vidrio...

\section{Revestimientos}

- Enfoscados, revocos; Guarnecidos; Paramentos de piedra, cerámicos, textiles, empapelados; Pinturas; Pavimentos; Techos...

c) Inclusión en el proyecto de cada edificio de un capítulo de Control de Calidad con memoria de ejecución, Pliego de Condiciones y presupuesto diferenciado con precios y mediciones para cada ensayo.

d) Realización de ensayos por laboratorios oficiales 0 bien homologados por la Administración, no vinculados al contratista y responsables única y exclusivamente ante el Director de Obra.

e) Inspecciones visuales y comprobaciones en obras por personal propio de la Administración integrado en una red de alcance provincial, regional y nacional que realiza los controles establecidos en las Normas Tecnológicas de la Edificación, que no requieren ensayos y se ocupa de la interpretación de éstos cuando le son entregados por los laboratorios.

f) Después de la construcción y durante el periodo de explotación del edificio se aplican unos manuales de operaciones de conservación que ayudan a unificar criterios para una mejor gestión de presupuestos.

\section{Conclusión}

Como resumen de la actividad de control de calidad en la Dirección General de Correos y Teleco-

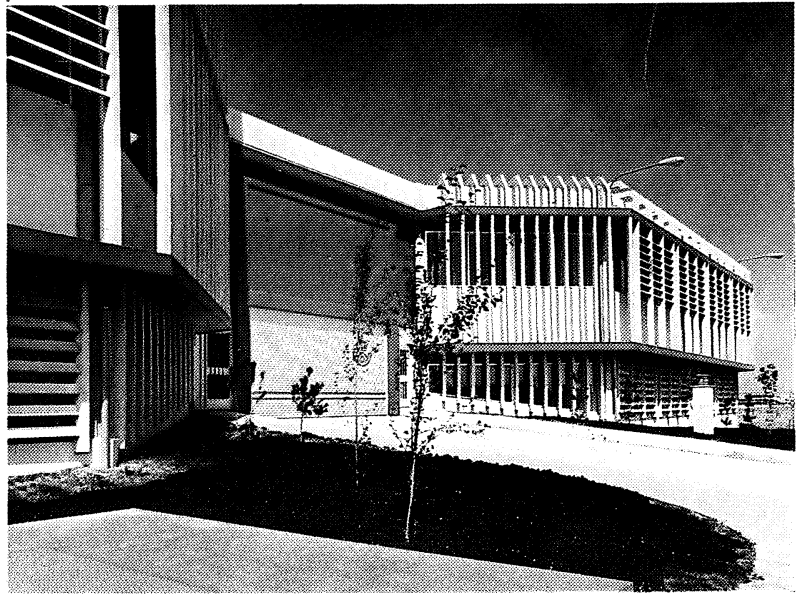

Centro de clasificación postal de Madrid, Barajas. (Arquitecto Antonio Moral.

municación, que se estima producirá un ahorro superior a los 4.700 millones de pesetas en 10 años, en gastos de inversión y conservación, puede decirse lo siguiente:

- Los factores más importantes de ahorro en inversiones son por este orden:

1) Aplicación de la "Guía de Control de Calidad" mediante presupuestos diferenciados para ensayos.

2) Realización de ensayos por laboratorios independientes del contratista.

3) Supervisión de la obra por los proyectistas.

4) Colaboración en la supervisión de obra por los técnicos encargados de su conservación, encuadrados en una organización nacional, regional y provincial.

- Los factores más importantes de ahorro en gastos de conservación son por este orden:

1) La actuación de los técnicos de cada localidad que permiten actuar a tiempo en cada problema con acciones preferentemente preventivas.

2) La realización de construcciones con mejor calidad.

3) El conocimiento de los problemas de cada obra, durante su construccion, por los técnicos encargados de su conservación.

4) La confección de manuales de uso, conservación y ahorro de energía.

Debido a la rentabilidad de los Sistemas de Control de Calidad creemos que su aplicación por parte de un sector de la Administración Española a la construcción y conservación de su patrimonio, mediante procedimientos prácticos como los descritos anteriormente, es una actividad que redunda en beneficio de todos a través de los ahorros en el presupuesto y el mejor servicio prestado por la infraestructura inmobiliaria. 